\title{
MODELING ERROR IN APPROXIMATE DECONVOLUTION MODELS*
}

\author{
ARGUS A. DUNCA ${ }^{\dagger}$ AND ROGER LEWANDOWSKI ${ }^{\ddagger}$
}

\begin{abstract}
We investigate the asymptotic behavior of the modeling error in 3D periodic Approximate Deconvolution Models, when the order $N$ of deconvolution goes to $\infty$. We consider generalized Helmholtz filters of order $p$, then the Gaussian filter. For Helmholtz filters, we estimate the rate of convergence to zero thanks to energy budgets, Gronwall's Lemma, and sharp inequalities applied to the Fourier coefficients of the residual stress. We next explain why the same analysis does not imply convergence to zero of the modeling error in the case of the Gaussian filter, leaving open issues.
\end{abstract}

Key words. Navier-Stokes equations, large eddy simulation, deconvolution models.

AMS subject classifications. 76D05, 35Q30, 76F65, 76D03.

\section{Introduction}

Direct numerical simulations of flows from the Navier-Stokes equations (NSE)

$$
\begin{aligned}
\mathbf{u}_{t}+\nabla \cdot(\mathbf{u} \otimes \mathbf{u})-\nu \Delta \mathbf{u}+\nabla p & =\mathbf{f}, \\
\nabla \cdot \mathbf{u} & =0, \\
\mathbf{u}(\mathbf{x}, 0) & =\mathbf{u}_{0}(\mathbf{x}),
\end{aligned}
$$

are accurate only for small Reynolds numbers. For large Reynolds numbers, flows are turbulent and only means or large scales of velocity and pressure fields may be computed via turbulence models.

The derivation of Large Eddy Simulation (LES) models of turbulent flows follows an application to the NSE of a low pass filter specified by a convolution kernel $G$, leading to the filtered NSE

$$
\begin{aligned}
\overline{\mathbf{u}}_{t}+\nabla \cdot(\overline{\mathbf{u}} \otimes \overline{\mathbf{u}})-\nu \Delta \overline{\mathbf{u}}+\nabla \bar{p} & =\overline{\mathbf{f}}+\nabla \cdot \mathbb{S}(\mathbf{u}, \mathbf{u}), \\
\nabla \cdot \overline{\mathbf{u}} & =0 \\
\overline{\mathbf{u}}(\mathbf{x}, 0) & =\overline{\mathbf{u}_{0}}(\mathbf{x}),
\end{aligned}
$$

where $\overline{\mathbf{u}}=G \star \mathbf{u}$ is the filtered velocity, $\bar{p}=G \star p$ the filtered pressure, and

$$
\mathbb{S}(\mathbf{u}, \mathbf{u})=\overline{\mathbf{u}} \otimes \overline{\mathbf{u}}-\overline{\mathbf{u} \otimes \mathbf{u}}
$$

is the subfilter scale stress tensor. A modeling process seeks suitable approximations of $\mathbb{S}(\mathbf{u}, \mathbf{u})$ in terms of $\overline{\mathbf{u}}$ in the scope of closing system (1.2). This yields, in the end, an LES model [5, 11, 33].

Most LES models are over-diffusive and tend to underestimate the energy, creating a subfilter scale region (SFS). The total error committed is the sum of the numerical error NE and the SFS area [7]. To reduce the SFS area, one may apply a deconvolution operator to the filter $[7,13,38,25,26]$.

*Received: October 30, 2012; accepted (in revised form): June 5, 2013. Communicated by Francois Golse.

${ }^{\dagger}$ Faculty of Mathematics and Computer Science, Spiru Haret University, Bucharest, 030045, Romania (a.a.dunca.mi@spiruharet.ro).

${ }^{\ddagger}$ IRMAR, UMR 6625, Université Rennes 1, Campus Beaulieu, 35042 Rennes cedex France (Roger.Lewandowski@univ-rennes1.fr, http://perso.univ-rennes1.fr/roger.lewandowski). 


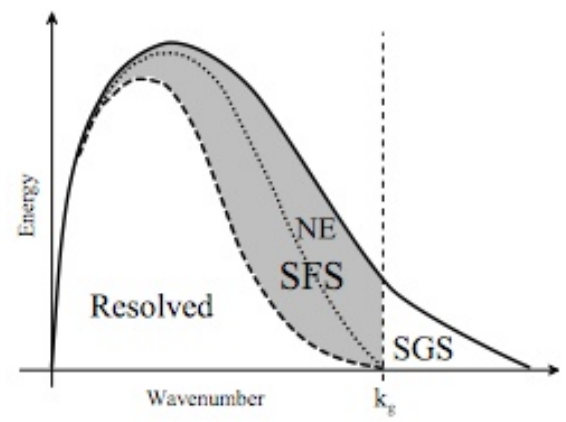

FIG. 1.1. From Chow et al. 2005 [7]. American Meteorological Society. Reprinted with permission.

In the case of the simplified Bardina model $[3,24,6]$, the deconvolution procedure is based on the approximation

$$
\mathbb{S}(\mathbf{u}, \mathbf{u}) \approx \mathbb{S}(\overline{\mathbf{u}}, \overline{\mathbf{u}})=\overline{\mathbf{u}} \otimes \overline{\mathbf{u}}-\overline{\overline{\mathbf{u}} \otimes \overline{\mathbf{u}}}
$$

The approximate deconvolution model (ADM in what follows) is derived from the simplified Bardina model by changing approximation (1.4) to

$$
\mathbb{S}(\mathbf{u}, \mathbf{u}) \approx \mathbb{S}_{N}(\overline{\mathbf{u}}, \overline{\mathbf{u}})=\overline{\mathbf{u}} \otimes \overline{\mathbf{u}}-\overline{D_{N}(\overline{\mathbf{u}}) \otimes D_{N}(\overline{\mathbf{u}})},
$$

leading to the ADM system below with solution $\left(\overline{\mathbf{u}}_{N}, \bar{p}_{N}\right)$ :

$$
\begin{aligned}
\partial_{t} \overline{\mathbf{u}}_{N}+\nabla \cdot\left(\overline{D_{N}\left(\overline{\mathbf{u}}_{N}\right) \otimes D_{N}\left(\overline{\mathbf{u}}_{N}\right)}\right)-\nu \Delta \overline{\mathbf{u}}_{N}+\nabla \bar{p}_{N} & =\overline{\mathbf{f}}, \\
\nabla \cdot \overline{\mathbf{u}}_{N} & =0, \\
\overline{\mathbf{u}}_{N}(0, \mathbf{x}) & =\overline{\mathbf{u}_{0}}(\mathbf{x}),
\end{aligned}
$$

where the deconvolution operator $D_{N}$ is given by the formula

$$
D_{N}=\sum_{n=0}^{N}(I-G)^{n} .
$$

Here, for simplicity, $G$ still denotes the operator associated with the kernel $G$. We always have $\mathbb{S}_{0}(\overline{\mathbf{u}}, \overline{\mathbf{u}})=\mathbb{S}(\overline{\mathbf{u}}, \overline{\mathbf{u}})$ and, if $\|G\|<1^{1}$, then, for a fixed $\mathbf{u}$,

$$
\lim _{N \rightarrow \infty} \mathbb{S}_{N}(\overline{\mathbf{u}}, \overline{\mathbf{u}})=\mathbb{S}(\mathbf{u}, \mathbf{u})
$$

The ADMs (1.6) shown above have been introduced by Stolz, Adams, and Kleiser in a series of papers $[2,39,40,37]$, showing very good performance of the ADM in the practical tests the above authors have carried out.

Following their work, in the last decade the ADMs have been the focus of intense research, both theoretical as well as computational; see [17, 19, 18, 6, 21, 20, $22,26,32,34,4,14,31]$. The ADM class of models has been enlarged to include

\footnotetext{
${ }^{1}$ The operator norm is based on natural energy spaces to which the fields belong, which will be latter specified.
} 
other deconvolution procedures as well, such as the Tichonov deconvolution method, [35], Chebishev optimized ADM [23], the continuous deconvolution method [28], or the multiscale deconvolution method [8]. In the next lines we list some of the mathematical properties of ADMs that have been proved in the above papers as well as the issues that are investigated in this report.

Existence and uniqueness of a solution to system (1.6) was proved in [9] when $G$ is the usual Helmholtz filter in the 3D periodic case, and in [4] for more general filters. More generally, if one can prove existence and uniqueness of a solution to system (1.6) for any $G$ that satisfies (1.8), it is expected that the sequence $\left(\overline{\mathbf{u}}_{N}, \bar{p}_{N}\right)_{N \in \mathbb{N}}$ converges to $(\overline{\mathbf{u}}, \bar{p})=(G \mathbf{u}, G p)$, for some solution $(\mathbf{u}, p)$ of the NSE [4].

Such convergence results have been proved in [4] in the 3D periodic case, when $G=G_{\alpha, p}$ is the generalized Helmholtz filter of order $p$ with $p \geq 3 / 4$, where

$$
G_{\alpha, p}(\mathbf{x})=\sum_{\mathbf{k} \in \mathcal{T}_{3}^{\star}} \frac{e^{i \mathbf{k} \cdot \mathbf{x}}}{1+\alpha^{2 p}|\mathbf{k}|^{2 p}},{ }^{2}
$$

upon proving existence and uniqueness of $\left(\overline{\mathbf{u}}_{N}, \bar{p}_{N}\right)$. In Definition (1.9), $\mathcal{T}_{3}:=2 \pi \mathbb{Z}^{3} / L$, $L>0$, denotes the size of the computational box and $\alpha>0$ is the filter's width, usually of the same magnitude as the mesh size in a numerical simulation (see [27] for further discussions). We should not be limited to the usual case $p=1$, as will be discussed later.

In this paper we investigate the remaining issue of the convergence rate of the modeling error $\boldsymbol{\varepsilon}_{N}=\overline{\mathbf{u}}-\overline{\mathbf{u}}_{N}$ to 0 in terms of $N$, as $N$ goes to infinity. Staying within the 3D periodic framework and the generalized Helmholtz filter of order $p(p \geq 3 / 4)$, we show in this paper that $L^{2}$ and $H^{1}$ norms of $\varepsilon_{N}$ are of order $(p(N+1))^{-1 / 4}$ (see our main result, Theorem 3.1 below).

To derive this rate of convergence, we first write the equation satisfied by $\varepsilon_{N}$, by subtracting (1.6) from (1.2),

$$
\partial_{t} \varepsilon_{N}+\nabla \cdot\left(\overline{D_{N} \varepsilon_{N} \otimes D_{N} \overline{\mathbf{u}}_{N}}\right)-\nu \Delta \varepsilon_{N}+\nabla r_{N}=-\nabla \cdot \overline{\boldsymbol{\tau}}_{N}-\nabla \cdot \overline{D_{N} \overline{\mathbf{u}} \otimes D_{N} \varepsilon_{N}},
$$

where $r_{N}=\bar{p}-\bar{p}_{N}$, and

$$
\boldsymbol{\tau}_{N}=\mathbf{u} \otimes \mathbf{u}-D_{N} \overline{\mathbf{u}} \otimes D_{N} \overline{\mathbf{u}}
$$

is the residual stress. By using successively an energy budget procedure and Gronwall's Lemma, we get an inequality satisfied by the norms of $A^{1 / 2} D_{N} \varepsilon_{N}$, where $A=G^{-1}$ (in terms of operators), from which we deduce an inequality satisfied by the norms of $\varepsilon_{N}$ itself (see inequality (3.30) below). This inequality highlights the role played by the $L^{2}$ norm of the residual stress.

The weakness of this approach is the regularity assumption imposed on the field $\mathbf{u}$, which is $L^{4}\left(0, T ; H^{1}(\Omega)\right)$. However, such assumptions are similar to the usual uniqueness proofs of the NSE.

To conclude, we have to estimate the $L^{2}$ norm of the residual stress (see inequality (4.9)). We carry out this calculation by using Fourier series expansions and calculations outlined in Appendix A, which, although using only elementary real analysis, are not straightforward and were first deduced and checked using numerical and symbolic computations with Octave and Maxima software packages [10, 1], before being rigorously proved.

\footnotetext{
${ }^{2}$ In terms of operators $G_{\alpha, p}=\left(\mathrm{I}-\alpha^{2 p} \Delta^{p}\right)^{-1}$, where $\Delta^{p}$ denotes the $p$-Laplacian, and $A_{\alpha, p}=$ $\mathrm{I}-\alpha^{2 p} \Delta^{p}=G^{-1}$.
} 
We observe that the rate of convergence slows down as $p$ increases in the range $[1, \infty[$. Moreover, the resulting bound goes to a constant that only depends on $\alpha$ and $\mathbf{u}$ when $p$ goes to infinity and $N$ remains fixed. This is consistent with the idea that the larger $p$, the smoother the filtered fields, enlarging the SFS area. Therefore, one needs a high order of deconvolution to reconstruct well the resolved scale area for large values of $p$.

Then we consider the popular Gaussian filter,

$$
\tilde{G}_{\alpha}(\mathbf{x})=\left(\frac{6}{\alpha^{2} \pi}\right)^{3 / 2} \exp \left(-\frac{6}{\alpha^{2}}\|\mathbf{x}\|^{2}\right)
$$

often used in LES. Applying the ADM theory for general abstract filters developed in [34] we deduce that the ADM is well-posed in the case of the Gaussian filter. Therefore, one may ask if there is convergence of the model to the filtered NSE when $N \rightarrow \infty$, and if yes, then what is the convergence rate?

The theory we develop for Helmholtz filters to determine the convergence rate of the modeling error in terms of $\mathrm{N}$ does not apply to the Gaussian filter because of the strong convergence of its Fourier modes to zero as the wave number increases, although this is not evidence that a convergence to zero in some weak sense is never possible.

Instead, we show that the Gaussian filter can be approximated by

$$
\tilde{G}_{\alpha, m}(\mathbf{x})=\sum_{\mathbf{k} \in \mathcal{T}_{3}}\left(1+\frac{\alpha^{2}|\mathbf{k}|^{2}}{24 m}\right)^{-m} e^{i \mathbf{k} \cdot \mathbf{x}, 3}
$$

when $m \rightarrow \infty$, and we prove that our procedure is still valid for this sequence of filters, and we derive a bound of order $(N+1)^{-4 m}$ for them. This bound goes to a constant depending on $\alpha$ and $\mathbf{u}$ when $m$ goes to infinity for a fixed $N$. Therefore, we cannot conclude that the deconvolution process converges to the filtered field $(\overline{\mathbf{u}}, \bar{p})$ in the case of the Gaussian filter. Because of the strong regularization effect of this filter, we may conjecture that if such a convergence would hold, then it should be very low. Therefore, the deconvolution process does not seem to be appropriate for the Gaussian filter. This remains an open issue.

The paper is organized as follows. We first fix the mathematical framework and recall the results of [4] useful for the remaining sections of the paper. We next detail how to bound the modeling error in terms of the residual stress for the Helmholtz filter and its powers. Further on, the $L^{2}$ norm of the residual stress is estimated by Fourier series expansions and inequalities presented in the appendix. We finally consider the Gaussian Filter. We show how to approximate it by the $G_{\alpha, m}$ 's, (1.13), and how to estimate the modeling error of the latter.

The paper ends with a series of remarks and open problems, mainly regarding the typical size of the constants involved in the inequalities (see Subsection 6.1). Indeed, these constants seem to be physically very large, hinting to some drawbacks of the method, although we conjecture the optimality of the convergence rate we have found in terms of $N$. Therefore, better constants should be achieved, yielding the main problem left open in our work.

A technical appendix includes key inequalities used to derive estimates of the residual stress.

\footnotetext{
${ }^{3}$ In terms of operators $\tilde{G}_{\alpha, m}=\left(1-\frac{\alpha^{2}}{24 m} \Delta\right)^{-m}$.
} 


\section{Mathematical framework}

2.1. Function spaces. Throughout the paper, $\nu>0$ and $\alpha>0$ are fixed and we stay within the periodic case framework. The domain of study is the $3 \mathrm{D}$ torus

$$
\mathbb{T}_{3}=\mathbb{R}^{3} / \mathcal{T}_{3}, \quad \text { where } \quad \mathcal{T}_{3}:=2 \pi \mathbb{Z}^{3} / L,
$$

for some given $L>0$, which is the size of the computational box. All the fields we consider have zero mean on $\mathbb{T}_{3}$. Let $\mathbb{H}_{s}$ be the vector field space

$$
\mathbb{H}_{s}=\left\{\mathbf{w}=\left(w_{1}, w_{2}, w_{3}\right)=\sum_{\mathbf{k} \in \mathcal{T}_{3}^{\star}} \widehat{\mathbf{w}}_{\mathbf{k}} e^{i \mathbf{k} \cdot \mathbf{x}}: \sum_{\mathbf{k} \in \mathcal{T}_{3}^{\star}}|\mathbf{k}|^{2 s}\left|\widehat{\mathbf{w}}_{\mathbf{k}}\right|^{2}<\infty\right\},
$$

equipped with the Hermitian structure defined by the inner product and its associated norm

$$
(\mathbf{w}, \mathbf{v})_{s}=\sum_{\mathbf{k} \in \mathcal{T}_{3}^{\star}}|\mathbf{k}|^{2 s} \widehat{\mathbf{w}}_{\mathbf{k}} \cdot \widehat{\mathbf{v}}_{\mathbf{k}}^{\star}, \quad\|\mathbf{w}\|_{s}=\left(\sum_{\mathbf{k} \in \mathcal{T}_{3}^{\star}}|\mathbf{k}|^{2 s}\left|\mathbf{w}_{\mathbf{k}}\right|^{2}\right)^{\frac{1}{2}},
$$

where

$$
\forall \mathbf{k}=\left(k_{1}, k_{2}, k_{3}\right) \in \mathcal{T}_{3}, \quad|\mathbf{k}|^{2}=k_{1}^{2}+k_{2}^{2}+k_{3}^{2},
$$

and $z^{\star}$ denotes the complex conjugate of $z$. It can be proved (see [29]) that for all $s \in \mathbb{R}$

$$
\mathbb{H}_{s} \text { is isomorphic to } H^{s}\left(\mathbb{T}_{3}\right)^{3}, \quad\left(\mathbb{H}_{s}\right)^{\prime}=\mathbb{H}_{-s},
$$

and we denote by

$$
\forall(\mathbf{w}, \mathbf{v}) \in \mathbb{H}_{-s} \times \mathbb{H}_{s}, \quad{ }_{-s}(\mathbf{w}, \mathbf{v})_{s}=\sum_{\mathbf{k} \in \mathcal{T}_{3}^{\star}} \widehat{\mathbf{w}}_{\mathbf{k}} \cdot \widehat{\mathbf{v}}_{\mathbf{k}}^{\star}
$$

the duality pairing.

Let $\mathbf{H}_{s} \subset \mathbb{H}_{s}$ be the closed subspace of fields valued in $\mathbb{R}^{3}$, characterized by

$$
\mathbf{H}_{s}=\left\{\mathbf{w}=\sum_{\mathbf{k} \in \mathcal{T}_{3}^{\star}} \widehat{\mathbf{w}}_{\mathbf{k}} e^{i \mathbf{k} \cdot \mathbf{x}} \in \mathbb{H}_{s}: \forall \mathbf{k} \in \mathcal{T}_{3}^{\star}, \quad \widehat{\mathbf{w}}_{\mathbf{k}}^{\star}=\widehat{\mathbf{w}}_{-\mathbf{k}} \text { and } \mathbf{k} \cdot \widehat{\mathbf{w}}_{\mathbf{k}}=0\right\} .
$$

One can show (see [29]) that

$$
\mathbf{H}_{s}=\left\{\mathbf{w}: \mathbb{T}_{3} \rightarrow \mathbb{R}^{3}, \mathbf{w} \in H^{s}\left(\mathbb{T}_{3}\right)^{3}, \quad \nabla \cdot \mathbf{w}=0, \quad \int_{\mathbb{T}_{3}} \mathbf{w} d \mathbf{x}=\mathbf{0}\right\} .
$$

\subsection{Operators.}

2.2.1. The filter. The general Helmholtz filter $\overline{\mathbf{w}}=G_{\alpha, p} \star \mathbf{w}$ is defined by the Fourier series expansion of the kernel $G_{\alpha, p}$ :

$$
G_{\alpha, p}(\mathbf{x})=\sum_{\mathbf{k} \in \mathcal{T}_{3}^{\star}} \widehat{G}_{\mathbf{k}} e^{i \mathbf{k} \cdot \mathbf{x}}, \quad \widehat{G}_{\mathbf{k}}=\frac{1}{1+\alpha^{2 p}|\mathbf{k}|^{2 p}} .
$$


Viewed as an operator, one has $G_{\alpha, p}=\left(\mathrm{I}-\alpha^{2 p} \Delta^{2 p}\right)^{-1}$. Furthermore, given a divergence free field $\mathbf{w}, \overline{\mathbf{w}}$ is solution of the PDE problem

$$
\begin{aligned}
-\alpha^{2 p} \Delta^{p} \overline{\mathbf{w}}+\overline{\mathbf{w}}+\nabla q & =\mathbf{w} \text { in } \mathbb{T}_{3}, \\
\nabla \cdot \overline{\mathbf{w}} & =0 \text { in } \mathbb{T}_{3},
\end{aligned}
$$

where the Lagrange multiplier $q$ is constant in this case.

From now on, we write $G$ instead of $G_{\alpha, p}$, and we denote in the same way the kernel and the operator. For all $s \geq 0, G$ defines an isomorphism,

$$
G:\left\{\mathbf{w}=\sum_{\mathbf{k} \in \mathcal{T}_{3}^{\star}} \widehat{\mathbf{w}}_{\mathbf{k}} e^{i \mathbf{k} \cdot \mathbf{x}} \longrightarrow \mathbb{H}_{s} \longrightarrow \overline{\mathbf{w}}=\sum_{\mathbf{k} \in \mathcal{T}_{3}^{\star}} \widehat{G}_{\mathbf{k}} \widehat{\mathbf{w}}_{\mathbf{k}} e^{i \mathbf{k} \cdot \mathbf{x}},\right.
$$

and we set $A=G^{-1}$, characterized by its kernel

$$
A(\mathbf{x})=\sum_{\mathbf{k} \in \mathcal{T}_{3}^{\star}} \widehat{A}_{\mathbf{k}} e^{i \mathbf{k} \cdot \mathbf{x}}, \quad \widehat{A}_{\mathbf{k}}=1+\alpha^{2 p}|\mathbf{k}|^{2 p} .
$$

Notice that if $\mathbf{w} \in \mathbf{H}_{s}$, then $\overline{\mathbf{w}} \in \mathbf{H}_{s+2 p}$ and the restriction of $G$ to $\mathbf{H}_{s}$, still denoted by $G$, is an isomorphism that maps $\mathbf{H}_{s}$ onto $\mathbf{H}_{s+2 p}$.

2.2.2. The deconvolution operators. Let $D_{N}$ denote the Van Cittert deconvolution operator, characterized by the kernel

$$
D_{N}=\sum_{0 \leq n \leq N}(\mathbf{I}-G)^{n}=\sum_{\mathbf{k} \in \mathcal{T}_{3}} \widehat{D}_{N, \mathbf{k}} e^{i \mathbf{k} \cdot \mathbf{x}}
$$

where

$$
\begin{aligned}
& \widehat{D}_{N, \mathbf{k}}=\sum_{n=0}^{N}\left(\frac{\alpha^{2 p}|\mathbf{k}|^{2 p}}{1+\alpha^{2 p}|\mathbf{k}|^{2 p}}\right)^{n}=\left(1+\alpha^{2 p}|\mathbf{k}|^{2 p}\right) \rho_{N, p, \mathbf{k}}, \\
& \rho_{N, p, \mathbf{k}}=1-\left(\frac{\alpha^{2 p}|\mathbf{k}|^{2 p}}{1+\alpha^{2 p}|\mathbf{k}|^{2 p}}\right)^{N+1} .
\end{aligned}
$$

The following holds [4]:

$$
\begin{aligned}
& 1 \leq \widehat{D}_{N, \mathbf{k}} \leq N+1, \quad \forall \mathbf{k} \in \mathcal{T}_{3}, \\
& \widehat{D}_{N, \mathbf{k}} \approx(N+1) \frac{1+\alpha^{2 p}|\mathbf{k}|^{2 p}}{\alpha^{2 p}|\mathbf{k}|^{2 p}}, \quad \text { for large }|\mathbf{k}|, \\
& \lim _{|\mathbf{k}| \rightarrow+\infty} \widehat{D}_{N, \mathbf{k}}=N+1, \\
& \widehat{D}_{N, \mathbf{k}} \leq\left(1+\alpha^{2 p}|\mathbf{k}|^{2 p}\right)=\widehat{A}_{\mathbf{k}}, \quad \forall \mathbf{k} \in \mathcal{T}_{3},
\end{aligned}
$$

where $\widehat{A}_{\mathbf{k}}$ is defined by (2.10). We deduce the following from (2.12) and (2.14).

Lemma 2.1. Given a real number $s \geq 0$, the operator $D_{N}$ is an isomorphism over $\mathbb{H}_{s}$, such that $1 \leq\left\|D_{N}\right\| \leq N+1$. Moreover, the subspace of divergence free fields $\mathbf{H}_{s}$ is stable under the action of $D_{N}$. 
2.3. Former results. This section presents some results proved in [4] with regard to the system

$$
\begin{array}{r}
\partial_{t} \overline{\mathbf{u}}_{N}+\nabla \cdot\left(\overline{D_{N}\left(\overline{\mathbf{u}}_{N}\right) \otimes D_{N}\left(\overline{\mathbf{u}}_{N}\right)}\right)-\nu \Delta \overline{\mathbf{u}}_{N}+\nabla \bar{p}_{N}=\overline{\mathbf{f}}, \\
\nabla \cdot \overline{\mathbf{u}}_{N}=0, \\
\overline{\mathbf{u}}_{N}(0, \mathbf{x})=\overline{\mathbf{u}}_{0}(\mathbf{x}) .
\end{array}
$$

Throughout the paper, we assume that $\mathbf{u}_{0}$ and $\mathbf{f}$ satisfy

$$
\mathbf{u}_{0} \in \mathbf{H}_{0}, \quad \mathbf{f} \in L^{2}\left([0, T], H_{-1}\left(\mathbb{T}_{3}\right)\right)^{3},
$$

and $\alpha>0$ is fixed.

Definition 2.2 (Regular Weak solution). We say that the couple $\left(\overline{\mathbf{u}}_{N}, \bar{p}_{N}\right)$ is a "regular weak solution" of the system (2.16) if and only if the three following items are satisfied:

1. REgularity

$$
\begin{aligned}
& \overline{\mathbf{u}}_{N} \in L^{2}\left([0, T] ; \mathbf{H}_{1+p}\right) \cap C\left([0, T] ; \mathbf{H}_{p}\right), \\
& \partial_{t} \overline{\mathbf{u}}_{N} \in L^{2}\left([0, T] ; \mathbf{H}_{0}\right), \\
& \bar{p}_{N} \in L^{2}\left([0, T] ; H^{1}\left(\mathbb{T}_{3}\right)\right)
\end{aligned}
$$

\section{INITIAL DATA}

$$
\lim _{t \rightarrow 0}\left\|\overline{\mathbf{u}}_{N}(t, \cdot)-\overline{\mathbf{u}_{0}}\right\|_{\mathbf{H}_{p}}=0
$$

\section{WEAK FORMULATION}

$$
\begin{aligned}
& \forall \mathbf{v} \in L^{2}\left([0, T] ; H^{1}\left(\mathbb{T}_{3}\right)^{3}\right) \\
& \int_{0}^{T} \int_{\mathbb{T}_{3}} \partial_{t} \overline{\mathbf{u}}_{N} \cdot \mathbf{v}-\int_{0}^{T} \int_{\mathbb{T}_{3}} \overline{D_{N}\left(\overline{\mathbf{u}}_{N}\right) \otimes D_{N}\left(\overline{\mathbf{u}}_{N}\right)}: \nabla \mathbf{v} \\
& \quad+\nu \int_{0}^{T} \int_{\mathbb{T}_{3}} \nabla \overline{\mathbf{u}}_{N}: \nabla \mathbf{v}+\int_{0}^{T} \int_{\mathbb{T}_{3}} \nabla \bar{p}_{N} \cdot \mathbf{v}=\int_{0}^{T} \int_{\mathbb{T}_{3}} \overline{\mathbf{f}} \cdot \mathbf{v} .
\end{aligned}
$$

Theorem 2.3. ([4]) Assume $p \geq 3 / 4$. Problem (2.16) has a unique regular weak solution. Moreover, when $p \geq 1$,

$$
\partial_{t} \overline{\mathbf{u}}_{N} \in L^{2}\left([0, T], \mathbf{H}_{p-1}\right), \quad \bar{p}_{N} \in L^{2}\left([0, T], H^{p}\left(\mathbb{T}_{3}\right)\right) .
$$

Theorem 2.4. ([4]) Assume $\mathbf{f} \in L^{2}\left([0, T] \times \mathbb{T}_{3}\right)^{3}$. There exists a weak dissipative solution of the NSE (1.1)

$$
(\mathbf{u}, p) \in\left[L^{2}\left([0, T], \mathbf{H}_{1}\right) \cap L^{2}\left([0, T], \mathbf{H}_{0}\right)\right] \times L^{5 / 3}\left([0, T] \times \mathbb{T}_{3}\right)
$$

such that from the sequence $\left(\overline{\mathbf{u}}_{N}, \bar{p}_{N}\right)_{N \in \mathbb{N}}$, one can extract a sub-sequence (still denoted $\left.\left(\overline{\mathbf{u}}_{N}, \bar{p}_{N}\right)_{N \in \mathbb{N}}\right)$ such that

$$
\begin{aligned}
& \overline{\mathbf{u}}_{N} \rightarrow \overline{\mathbf{u}}\left\{\begin{array}{l}
\text { weakly in } L^{2}\left([0, T], \mathbf{H}_{1+p}\left(\mathbb{T}_{3}\right)^{3}\right) \cap L^{\infty}\left([0, T], \mathbf{H}_{p}\right), \\
\text { strongly in } L^{r}\left([0, T] ; H^{p}\left(\mathbb{T}_{3}\right)^{3}\right), \quad \forall 1 \leq r<+\infty,
\end{array}\right. \\
& \bar{p}_{N} \rightarrow \bar{p} \text { weakly in } L^{2}\left([0, T] ; H^{1}\left(\mathbb{T}_{3}\right)\right) \cap L^{5 / 3}\left([0, T] ; W^{2 p, 5 / 3}\left(\mathbb{T}_{3}\right)\right) .
\end{aligned}
$$




\section{The estimate of the modeling error}

3.1. The regularity assumption and the main result. Let $\left(\overline{\mathbf{u}}_{N}, \bar{p}_{N}\right)$ be the solution of Problem (2.16). We assume that the limit $(\overline{\mathbf{u}}, \bar{p})=(G \mathbf{u}, G p)$ of $\left(\overline{\mathbf{u}}_{N}, \bar{p}_{N}\right)_{N \in \mathbb{N}}$ satisfies the regularity assumption

$$
\mathbf{u}=A \overline{\mathbf{u}} \in L^{4}\left(\mathbf{H}_{1}\right){ }^{4}
$$

By the Sobolev Embedding Theorem, we deduce

$$
\mathbf{u} \in L^{4}\left([0, T] \times \mathbb{T}_{3}\right)
$$

Because $(\mathbf{u}, p)$ is the solution of the NSE, one has

$$
\Delta p=-\nabla \cdot(\nabla \cdot(\mathbf{u} \otimes \mathbf{u}))+\nabla \cdot \mathbf{f},
$$

which yields in the periodic case

$$
p \in L^{2}([0, T] \times \Omega),
$$

and we derive from the NSE

$$
\partial_{t} \mathbf{u} \in L^{2}\left([0, T], \mathbf{H}_{-1}\right)
$$

Our main result is as follows.

TheOREM 3.1. Let $\boldsymbol{\varepsilon}_{N}=\overline{\mathbf{u}}-\overline{\mathbf{u}}_{N}$ be the modeling error, and assume that (3.1) holds. Then we have

$$
\begin{aligned}
& \left\|\varepsilon_{N}(t, \cdot)\right\|_{0}^{2}+\alpha^{2 p}\left\|\varepsilon_{N}(t, \cdot)\right\|_{p}^{2}+\nu \int_{0}^{t}\left(\left\|\nabla \varepsilon_{N}(s, \cdot)\right\|_{0}^{2}+\alpha^{2 p}\left\|\nabla \varepsilon_{N}(s, \cdot)\right\|_{p}^{2}\right) d s \\
\leq & \frac{8 C^{2} \alpha}{\nu(2 p(N+1))^{1 / 2 p}}\|\mathbf{u}\|_{L^{4}\left(\mathbf{H}_{1}\right)}^{4} e^{\frac{27}{2 \nu^{3}}\|\mathbf{u}\|_{L^{4}\left(\mathbf{H}_{1}\right)}^{4}} .
\end{aligned}
$$

where $C$ is a universal constant, obtained as a product of Sobolev constants.

3.2. Modeling error and residual stress. Let $\varepsilon_{N}$ and $\boldsymbol{\tau}_{N}$ be the modeling error and the residual stress defined by

$$
\begin{aligned}
& \varepsilon_{N}=\overline{\mathbf{u}}-\overline{\mathbf{u}}_{N}, \\
& \boldsymbol{\tau}_{N}=\mathbf{u} \otimes \mathbf{u}-D_{N} \overline{\mathbf{u}} \otimes D_{N} \overline{\mathbf{u}} .
\end{aligned}
$$

The equation satisfied by $\varepsilon_{N}$ is derived by subtracting (2.16) from the filtered NSE (1.2). Expressing the right hand side in terms of $\boldsymbol{\tau}_{N}$, we obtain

$$
\partial_{t} \varepsilon_{N}+\nabla \cdot\left(\overline{D_{N} \varepsilon_{N} \otimes D_{N} \overline{\mathbf{u}}_{N}}\right)-\nu \Delta \varepsilon_{N}+\nabla r_{N}=-\nabla \cdot \overline{\boldsymbol{\tau}}_{N}-\nabla \cdot\left(\overline{D_{N} \overline{\mathbf{u}} \otimes D_{N} \varepsilon_{N}}\right),
$$

where $r_{N}=\bar{p}-\bar{p}_{N}$.

The aim of this section is to estimate $\varepsilon_{N}$ in terms of $\boldsymbol{\tau}_{N}$. One may try to estimate first $A^{1 / 2} D_{N}^{1 / 2} \varepsilon_{N}$ rather than $\varepsilon_{N}$, because the natural multiplier to get an energy balance from equation (3.8) is $A D_{N} \varepsilon_{N}$, and formally $\left(\partial_{t} \varepsilon_{N}, A D_{N} \varepsilon_{N}\right)=$ $(d / 2 d t)\left\|A^{1 / 2} D_{N}^{1 / 2} \varepsilon_{N}\right\|_{0}$. Once $A^{1 / 2} D_{N}^{1 / 2} \varepsilon_{N}$ is estimated, we derive bounds for $\varepsilon_{N}$ (Corollary 3.1 below) by comparing the norms of the various operators.

\footnotetext{
${ }^{4}$ For simplicity, we use the notation $L^{4}\left(\mathbf{H}_{1}\right)$ instead of $L^{4}\left([0, T], \mathbf{H}_{1}\right)$.
} 
THEOREM 3.2. The following inequality holds for all $N>0$ and $t \geq 0$ :

$$
\begin{aligned}
& \left\|A^{1 / 2} D_{N}^{1 / 2} \varepsilon_{N}(t, \cdot)\right\|_{0}^{2}+\nu \int_{0}^{t}\left\|A^{1 / 2} D_{N}^{1 / 2} \varepsilon_{N}(s, \cdot)\right\|_{1}^{2} d s \\
\leq & \frac{2}{\nu} e^{\frac{27}{2 \nu^{3}}\|\mathbf{u}\|_{L^{4}}^{4}\left(\mathbf{H}_{1}\right)} \int_{0}^{t}\left\|\boldsymbol{\tau}_{N}(s, \cdot)\right\|_{0}^{2} d s .
\end{aligned}
$$

Proof. The proof is based on an energy equality satisfied by $A^{\frac{1}{2}} D_{N}^{\frac{1}{2}} \varepsilon_{N}$, to which one applies Gronwall's Lemma. To do so, we use $A D_{N} \varepsilon_{N}$ as a multiplier in (3.8), and then integrate by parts.

The proof is divided into three steps. In the first one, we check that $A D_{N} \varepsilon_{N}$ is appropriate as a test function. Next, we perform integrations by parts. In the last step, we apply a standard interpolation inequality in order to apply Gronwall's Lemma.

Step 3.i. Consistency of the procedure. We check the regularity of $A^{1 / 2} D_{N}^{1 / 2} \varepsilon_{N}$ and each factor in equation (3.8) one by one, beginning with $\varepsilon_{N}$. The regularity assumption (3.1) combined with the regularization effect (2.9) of the operator G gives $\overline{\mathbf{u}} \in L^{4}\left([0, T], \mathbf{H}_{1+2 p}\right)$. Therefore we have, from (2.19),

$$
\varepsilon_{N} \in L^{2}\left([0, T], \mathbf{H}_{1+p}\right) \subset L^{2}\left([0, T], \mathbb{H}_{1+p}\right) .
$$

Applying Lemma 2.1 combined with (2.9), we get

$$
A D_{N} \varepsilon_{N} \in L^{2}\left([0, T], \mathbb{H}_{1-p}\right) .
$$

We now prove that each factor in equation (3.8) is in

$$
L^{2}\left([0, T], \mathbb{H}_{p-1}\right)=\left(L^{2}\left([0, T], \mathbb{H}_{1-p}\right)\right)^{\prime}
$$

(see Subsection 2.1). For simplicity, we write things as

$$
\left.\begin{array}{rl}
(2.19) & +(3.5) \\
& +(2.24)
\end{array}\right\} \Rightarrow\left\{\begin{array}{lr}
\partial_{t} \varepsilon_{N} \in L^{2}\left([0, T], \mathbf{H}_{0}\right) & \text { if } 3 / 4 \leq p \leq 1 \\
\partial_{t} \varepsilon_{N} \in L^{2}\left([0, T], \mathbf{H}_{p-1}\right) & \text { if } p \geq 1
\end{array}\right.
$$

When $3 / 4 \leq p \leq 1, \mathbf{H}_{0} \hookrightarrow \mathbb{H}_{0} \hookrightarrow \mathbb{H}_{p-1}$, and when $p \geq 1, \mathbf{H}_{p-1} \hookrightarrow \mathbb{H}_{p-1}$. In all cases,

$$
\partial_{t} \varepsilon_{N} \in L^{2}\left([0, T], \mathbb{H}_{p-1}\right) .
$$

Similarly,

$$
\left.\begin{array}{rl}
(2.20)+(3.4) \\
+(2.24)
\end{array}\right\} \Rightarrow\left\{\begin{array}{l}
\nabla r_{N} \in L^{2}\left([0, T] \times \mathbb{T}_{3}\right)^{3} \quad \text { if } 3 / 4 \leq p \leq 1 \\
\nabla r_{N} \in L^{2}\left([0, T], H^{p-1}\left(\mathbb{T}_{3}\right)^{3}\right)
\end{array}\right.
$$

which yields

$$
\nabla r_{N} \in L^{2}\left([0, T], \mathbb{H}_{p-1}\right) .
$$

From the embedding $\mathbf{H}_{1} \hookrightarrow \mathbb{H}_{1}$, we deduce

$$
(3.10) \Rightarrow \Delta \varepsilon_{N} \in L^{2}\left([0, T], \mathbb{H}_{p-1}\right) .
$$

Furthermore, as $(\mathbf{u}, p)$ is a dissipative solution to the NSE, $\mathbf{u} \in L^{\infty}\left([0, T], \mathbb{H}_{0}\right)$. Therefore $\overline{\mathbf{u}} \in L^{\infty}\left([0, T], \mathbb{H}_{2 p}\right)$, and by Lemma 2.1 we get

$$
D_{N} \overline{\mathbf{u}} \in L^{\infty}\left([0, T], \mathbb{H}_{2 p}\right),
$$


from which we conclude

$$
\left.\begin{array}{rr}
(2.18) & +\quad(3.17) \\
& + \text { Lemma } 2.1
\end{array}\right\} \Rightarrow D_{N} \varepsilon_{N} \in L^{\infty}\left([0, T], \mathbb{H}_{p}\right) .
$$

Because $p \geq 3 / 4$, we deduce from the Sobolev Embedding Theorem $\mathbf{H}_{p} \hookrightarrow L^{4}\left(\mathbb{T}_{3}\right)^{3}$ that

$$
\left.\begin{array}{r}
(3.17)+(3.18) \\
+(2.9)
\end{array}\right\} \Rightarrow \nabla \cdot\left(\overline{D_{N} \varepsilon_{N} \otimes D_{N} \overline{\mathbf{u}}_{N}}\right) \in L^{\infty}\left([0, T], \mathbb{H}_{2 p-1}\right) .
$$

Similarly,

$$
\nabla \cdot\left(\overline{D_{N} \overline{\mathbf{u}} \otimes D_{N} \varepsilon_{N}}\right) \in L^{\infty}\left([0, T], \mathbb{H}_{2 p-1}\right) .
$$

Finally, $\mathbf{u} \in L^{\infty}\left([0, T], \mathbb{H}_{0}\right)$ combined with (3.2) and properties of $G$ and $D_{N}$ given above, yields

$$
\nabla \cdot \bar{\tau}_{N} \in L^{2}\left([0, T], \mathbb{H}_{2 p-1}\right) .
$$

Bringing together all of these results, we conclude that if

$$
\mathbb{A}_{N}=\partial_{t} \varepsilon_{N}+\nabla \cdot\left(\overline{D_{N} \varepsilon_{N} \otimes D_{N} \overline{\mathbf{u}}_{N}}\right)-\nu \Delta \varepsilon_{N}+\nabla r_{N}+\nabla \cdot \overline{\boldsymbol{\tau}}_{N}+\nabla \cdot\left(\overline{D_{N} \overline{\mathbf{u}} \otimes D_{N} \varepsilon_{N}}\right),
$$

then $\mathbb{A}_{N} \in L^{2}\left([0, T], \mathbb{H}_{p-1}\right)$. Therefore, the duality pairing ${ }_{p-1}\left(\mathbb{A}_{N}, A D_{N} \varepsilon_{N}\right)_{1-p}$ is well defined, which makes consistent the multiplication of equation (3.8) by $A D_{N} \varepsilon_{N}$. In what follows, we omit the subscripts when writing duality pairings.

Step 3.ii. Energy equality. Because all the operators we consider are self adjoint, the following holds (see [30]):

$$
\begin{aligned}
& \left(\partial_{t} \varepsilon_{N}, A D_{N} \varepsilon_{N}\right)=\frac{d}{2 d t}\left\|A^{\frac{1}{2}} D_{N}^{\frac{1}{2}} \varepsilon_{N}\right\|_{0}^{2} \\
& \left(-\Delta \varepsilon_{N}, A D_{N} \varepsilon_{N}\right)=\left\|A^{\frac{1}{2}} D_{N}^{\frac{1}{2}} \varepsilon_{N}\right\|_{1}^{2} .
\end{aligned}
$$

Furthermore, because $A D_{N} \varepsilon_{N}$ has zero divergence, $\left(\nabla r_{N}, A D_{N} \varepsilon_{N}\right)=0$. Finally, as the operators commute with the differential operators,

$$
\begin{aligned}
& \left(\nabla \cdot\left(\overline{D_{N} \varepsilon_{N} \otimes D_{N} \mathbf{w}_{N}}\right), A D_{N} \boldsymbol{\varepsilon}_{N}\right)=\left(A^{-1} \nabla \cdot\left(D_{N} \varepsilon_{N} \otimes D_{N} \mathbf{w}_{N}\right), A D_{N} \varepsilon_{N}\right) \\
= & \left(A^{-1} \nabla \cdot\left(D_{N} \varepsilon_{N} \otimes D_{N} \mathbf{w}_{N}\right), A D_{N} \varepsilon_{N}\right)=\left(\nabla \cdot\left(D_{N} \varepsilon_{N} \otimes D_{N} \mathbf{w}_{N}\right), D_{N} \varepsilon_{N}\right) \\
= & \left(\left(D_{N} \mathbf{w}_{N} \cdot \nabla\right) D_{N} \varepsilon_{N}, D_{N} \varepsilon_{N}\right)=0,
\end{aligned}
$$

because $D_{N} \mathbf{w}_{N}$ has zero divergence. Finally, arguing as in (3.23) to eliminate the bar in the integrals of the right hand side, we get

$$
\frac{d}{2 d t}\left\|A^{\frac{1}{2}} D_{N}^{\frac{1}{2}} \varepsilon_{N}\right\|_{0}^{2}+\nu\left\|A^{\frac{1}{2}} D_{N}^{\frac{1}{2}} \varepsilon_{N}\right\|_{1}^{2}=\left(\boldsymbol{\tau}_{N}, \nabla D_{N} \varepsilon_{N}\right)-\left(\left(D_{N} \varepsilon_{N} \cdot \nabla\right) D_{N} \overline{\mathbf{u}}, D_{N} \varepsilon_{N}\right) .
$$

Step 3.iii. Bounds and Gronwall's Lemma. We bound each term of the right hand side of (3.24) one by one. From the Cauchy-Schwarz inequality combined with Young inequality, we get

$$
\left|\left(\boldsymbol{\tau}_{N}, \nabla D_{N} \varepsilon_{N}\right)\right| \leq \frac{1}{\nu}\|\boldsymbol{\tau}\|_{0}^{2}+\frac{\nu}{4}\left\|D_{N} \varepsilon_{N}\right\|_{1}^{2} .
$$


In the same way, by using the Ladyzhenskaya's inequality [41] we obtain

$$
\begin{aligned}
& \left|\left(\left(D_{N} \varepsilon_{N} \cdot \nabla\right) D_{N} \overline{\mathbf{u}}, D_{N} \varepsilon_{N}\right)\right| \leq\left\|D_{N} \varepsilon_{N}\right\|_{L^{4}}^{2}\left\|D_{N} \overline{\mathbf{u}}\right\|_{1} \\
\leq & \left\|D_{N} \varepsilon_{N}\right\|_{0}^{\frac{1}{2}}\left\|D_{N} \varepsilon_{N}\right\|_{1}^{\frac{3}{2}}\left\|D_{N} \overline{\mathbf{u}}\right\|_{1} .
\end{aligned}
$$

The symbol of $D_{N} G$ is equal to $\rho_{N, p, \mathbf{k}} \in[0,1]$ (see (2.11)). Therefore, we have $\left\|D_{N} \overline{\mathbf{u}}\right\|_{1} \leq\|\mathbf{u}\|_{1}$. By Young's inequality combined with (3.26), we obtain

$$
\left|\left(\left(D_{N} \boldsymbol{\varepsilon}_{N} \cdot \nabla\right) D_{N} \overline{\mathbf{u}}, D_{N} \varepsilon_{N}\right)\right| \leq \frac{27}{4 \nu^{3}}\|\mathbf{u}\|_{1}^{4}\left\|D_{N} \varepsilon_{N}\right\|_{0}^{2}+\frac{\nu}{4}\left\|D_{N} \varepsilon_{N}\right\|_{1}^{2} .
$$

We deduce from (2.15) that the symbol of $D_{N}$ is less than the symbol of $A^{1 / 2} D_{N}^{1 / 2}$, which leads to

$$
\left\|D_{N} \varepsilon_{N}\right\|_{0} \leq\left\|A^{\frac{1}{2}} D_{N}^{\frac{1}{2}} \varepsilon_{N}\right\|_{0}
$$

regardless of $N$. Combining (3.24), (3.25), (3.26), and (3.28) yields

$$
\frac{d}{d t}\left\|A^{\frac{1}{2}} D_{N}^{\frac{1}{2}} \varepsilon_{N}\right\|_{0}^{2}+\nu\left\|A^{\frac{1}{2}} D_{N}^{\frac{1}{2}} \varepsilon_{N}\right\|_{1}^{2} \leq \frac{2}{\nu}\|\boldsymbol{\tau}\|_{0}^{2}+\frac{27}{2 \nu^{3}}\|\mathbf{u}\|_{1}^{4}\left\|A^{\frac{1}{2}} D_{N}^{\frac{1}{2}} \varepsilon_{N}\right\|_{0}^{2}
$$

Inequality (3.9) results from inequality (3.29) thanks to a standard generalization of Gronwall's Lemma [12].

Corollary 3.1. The modeling error $\varepsilon_{N}$ satisfies

$$
\begin{aligned}
& \left\|\varepsilon_{N}(t, \cdot)\right\|_{0}^{2}+\alpha^{2 p}\left\|\varepsilon_{N}(t, \cdot)\right\|_{p}^{2}+\nu \int_{0}^{t}\left(\left\|\nabla \varepsilon_{N}(s, \cdot)\right\|_{0}^{2}+\alpha^{2 p}\left\|\nabla \varepsilon_{N}(s, \cdot)\right\|_{p}^{2}\right) d s \\
\leq & \frac{2}{\nu} e^{\frac{27}{2 \nu^{3}}\|\mathbf{u}\|_{L^{4}\left(\mathbf{H}_{1}\right)}^{4}} \int_{0}^{t}\left\|\boldsymbol{\tau}_{N}(s, \cdot)\right\|_{0}^{2} d s,
\end{aligned}
$$

for all $N>0$ and $t \geq 0$.

Proof. Let $\mathbf{v}=\sum_{\mathbf{k} \in \mathcal{T}_{3}} \hat{\mathbf{v}}_{\mathbf{k}} e^{i \mathbf{k} \cdot \mathbf{x}} \in \mathbb{H}_{p}$. We observe that

$$
\left\|A^{\frac{1}{2}} \mathbf{v}\right\|_{0}^{2}=\sum_{\mathbf{k} \in \mathcal{T}_{3}}\left(1+\alpha^{2 p}|\mathbf{k}|^{2 p}\right)\left|\hat{\mathbf{v}}_{\mathbf{k}}\right|^{2}=\|\mathbf{v}\|_{0}^{2}+\alpha^{2 p}\|\mathbf{v}\|_{p}^{2}
$$

We first take $\mathbf{v}=D_{N}^{1 / 2} \varepsilon_{N}$ in (3.31). By using (2.12), which yields the general formal inequality $\|\mathbf{w}\|_{s} \leq\left\|D_{N}^{1 / 2} \mathbf{w}\right\|_{s}$, we deduce that

$$
\left\|\varepsilon_{N}\right\|_{0}^{2}+\alpha^{2 p}\left\|\varepsilon_{N}\right\|_{p}^{2} \leq\left\|A^{1 / 2} D_{N}^{1 / 2} \varepsilon_{N}\right\|_{0}^{2} .
$$

We next take $\mathbf{v}=\partial_{i} D_{N}^{1 / 2} \varepsilon_{N}$ in (3.31), which yields

$$
\left\|\nabla \varepsilon_{N}\right\|_{0}^{2}+\alpha^{2 p}\left\|\nabla \varepsilon_{N}\right\|_{p}^{2} \leq\left\|A^{1 / 2} D_{N}^{1 / 2} \varepsilon_{N}\right\|_{1}^{2} .
$$

We deduce (3.30) from (3.9) thanks to (3.32) and (3.33). 


\section{Residual stress and rate of convergence}

Now that we have shown that the modeling error $\varepsilon_{N}$ is driven by the $L^{2}$ norm of the residual stress $\boldsymbol{\tau}_{N}$, involving the $L^{4}\left(\mathbf{H}_{1}\right)$ norm of $\mathbf{u}$, it remains to estimate the $L^{2}$ norm of $\boldsymbol{\tau}_{N}$, which is what we aim to carry out in this section. The framework, assumptions and notations are those of Section 3.

In what follows, $S_{s}$ denotes the Sobolev constant $^{5}$ in the embedding $\mathbb{H}_{s} \hookrightarrow$ $L^{s^{\star}}\left(\mathbb{T}_{3}\right)^{3}$. To begin, we show the following result.

Lemma 4.1. The following inequalities hold true:

$$
\begin{aligned}
& \left\|\boldsymbol{\tau}_{N}\right\|_{0} \leq 2 C\|\mathbf{u}(t, \cdot)\|_{1}\left\|\mathbf{u}-D_{N} \overline{\mathbf{u}}\right\|_{1 / 2}, \\
& \left\|\mathbf{u}-D_{N} \overline{\mathbf{u}}\right\|_{1 / 2}^{2} \leq \frac{\alpha}{(2 p(N+1))^{1 / 2 p}}\|\mathbf{u}\|_{1}^{2},
\end{aligned}
$$

where $C=S_{1} S_{1 / 2} \cdot{ }^{6}$

Proof. Step. 4.i. Proof of (4.1). We write $\boldsymbol{\tau}_{N}$ as

$$
\boldsymbol{\tau}_{N}=\left(\mathbf{u}-D_{N} \overline{\mathbf{u}}\right) \otimes \mathbf{u}+D_{N} \overline{\mathbf{u}} \otimes\left(\mathbf{u}-D_{N} \overline{\mathbf{u}}\right) .
$$

Therefore, combining Hölder's inequality with $1 / 3+1 / 6=1 / 2$ for conjugation and the Sobolev inequality $\|\mathbf{w}\|_{L^{6}} \leq S_{1}\|\mathbf{w}\|_{1}$, we get

$$
\|\boldsymbol{\tau}\|_{0} \leq 2 S_{1}\|\mathbf{u}\|_{1} \| \mathbf{u}-\left.D_{N} \overline{\mathbf{u}}\right|_{L^{3}\left(\mathbb{T}_{3}\right)^{3}},
$$

To estimate $\left\|\mathbf{u}-D_{N} \overline{\mathbf{u}}\right\|_{L^{3}\left(\mathbb{T}_{3}\right)^{3}}$, we use the embedding of $\mathbb{H}_{1 / 2}$ into $L^{3}\left(\mathbb{T}_{3}\right)^{3}$ to obtain

$$
\left\|\mathbf{u}-D_{N} \overline{\mathbf{u}}||_{L^{3}\left(\mathbb{T}_{3}\right)^{3}} \leq S_{1 / 2}|| \mathbf{u}-D_{N} \overline{\mathbf{u}}\right\|_{1 / 2},
$$

hence (4.1) follows by combining (4.4) and (4.5).

Step. 4.ii. Proof of (4.2). We deduce from (2.11) that

$$
\left\|\mathbf{u}-D_{N} \overline{\mathbf{u}}\right\|_{1 / 2}^{2}=\sum_{\mathbf{k} \in \mathcal{T}_{3}}\left(\frac{\alpha^{2 p}|\mathbf{k}|^{2 p}}{1+\alpha^{2 p}|\mathbf{k}|^{2 p}}\right)^{2(N+1)}|\mathbf{k}|\left|\hat{\mathbf{u}}_{\mathbf{k}}\right|^{2} .
$$

We apply the technical inequality (A.6) proved in Appendix A below, with $x=\alpha^{p}|\mathbf{k}|^{p}$, $a=2 p(N+1)>1$, which yields

$$
\left(\frac{\alpha^{2 p}|\mathbf{k}|^{2 p}}{1+\alpha^{2 p}|\mathbf{k}|^{2 p}}\right)^{2 p(N+1)} \leq \frac{\alpha^{p}|\mathbf{k}|^{p}}{\sqrt{2 p(N+1)}}
$$

We raise both sides of (4.7) to the power $1 / p$, multiply the result by $|\mathbf{k}|\left|\hat{\mathbf{u}}_{\mathbf{k}}\right|^{2}$ and get

$$
\left(\frac{\alpha^{2 p}|\mathbf{k}|^{2 p}}{1+\alpha^{2 p}|\mathbf{k}|^{2 p}}\right)^{2(N+1)}|\mathbf{k}|\left|\hat{\mathbf{u}}_{\mathbf{k}}\right|^{2} \leq \frac{\alpha}{(2 p(N+1))^{1 / 2 p}}|\mathbf{k}|^{2}\left|\hat{\mathbf{u}}_{\mathbf{k}}\right|^{2},
$$

hence (4.2) follows from (4.6).

\footnotetext{
${ }^{5}$ The constants $S_{1}$ and $S_{1 / 2}$ do not depend on $L$. One can prove that $S_{1} \leq(16+3 / \pi)^{1 / 3}$; see [29]. Unfortunately, we do not know a numerical bound for $S_{1 / 2}$, although it is likely that such a bound may be found in the literature

${ }^{6}$ Inequalities (4.1) and (4.2) both hold at any fixed time $t \in[0, T]$, which is not indicated here to simplify the notations.
} 
Corollary 4.2. The following estimate holds for all $t \in[0, T]$ :

$$
\left\|\boldsymbol{\tau}_{N}(t, \cdot)\right\|_{0}^{2} \leq \frac{(2 C)^{2} \alpha}{(2 p(N+1))^{1 / 2 p}}\|\mathbf{u}(t, \cdot)\|_{1}^{4} .
$$

Inequality (4.9) results from (4.2) combined with (4.1).

Summarizing: $(3.30)+(4.9) \Rightarrow$

$$
\begin{aligned}
& \left\|\varepsilon_{N}(t, \cdot)\right\|_{0}^{2}+\alpha^{2 p}\left\|\varepsilon_{N}(t, \cdot)\right\|_{p}^{2}+\nu \int_{0}^{t}\left(\left\|\nabla \varepsilon_{N}(s, \cdot)\right\|_{0}^{2}+\alpha^{2 p}\left\|\nabla \varepsilon_{N}(s, \cdot)\right\|_{p}^{2}\right) d s \\
\leq & \frac{8 C^{2} \alpha}{\nu(2 p(N+1))^{1 / 2 p}}\|\mathbf{u}\|_{L^{4}\left(\mathbf{H}_{1}\right)}^{4} e^{\frac{27}{2 \nu^{3}}\|\mathbf{u}\|_{L^{4}\left(\mathbf{H}_{1}\right)}^{4},}
\end{aligned}
$$

for all $N>0$ and $t \geq 0$.

\section{The case of the Gaussian filter}

5.1. Framework. In the scale space analysis (see A. Witkin [43]), structures are represented as families of their averages on different length scales. Furthermore, in the scale-space theory such families should fulfill certain scale-space axioms. In this respect, the generic (most used) scale-space is the linear Gaussian scale-space in which the smoothed family is obtained by convolving the structure under study with the Gaussian kernel $[42,15]$.

In the context of LES, Gaussian filtering has been used for flow representations at different length scales starting with the Bardina model [3], being one of the most popular filters appearing in the LES literature.

The Gaussian filter is specified by its kernel,

$$
\tilde{G}_{\alpha}(\mathbf{x})=\tilde{G}(\mathbf{x})=\left(\frac{6}{\alpha^{2} \pi}\right)^{3 / 2} \exp \left(-\frac{6}{\alpha^{2}}\|\mathbf{x}\|^{2}\right)
$$

where we omit the subscript $\alpha$ for simplicity. It can be shown (see [36]) that

$$
\tilde{G}(\mathbf{x})=\sum_{\mathbf{k} \in \mathcal{T}_{3}} \tilde{G}_{\mathbf{k}} e^{i \mathbf{k} \cdot \mathbf{x}}, \quad \text { where } \quad \tilde{G}_{\mathbf{k}}=e^{-\frac{\alpha^{2}|\mathbf{k}|^{2}}{24}} .
$$

Let $s \geq 0$ and $q \geq s$. There exists a constant $C$ such that

$$
\forall \mathbf{k} \in \mathcal{T}_{3}^{\star}, \quad \tilde{G}_{\mathbf{k}}|\mathbf{k}|^{q} \leq C|\mathbf{k}|^{s} .
$$

Therefore,

$$
\forall s \geq 0, \quad \forall \mathbf{u} \in \mathbb{H}_{s}, \quad \forall q \geq s, \quad \tilde{G} \mathbf{u} \in \mathbb{H}_{q} .
$$

Let $\mathbf{u}$ be given such that $\forall \mathbf{k} \in \mathcal{T}_{3}^{\star},\left|\hat{\mathbf{u}}_{\mathbf{k}}\right|=|\mathbf{k}|^{-1-q} \neq 0(q \geq 0)$. Such a vector field $\mathbf{u}$ belongs to $\mathbb{H}_{q}$, but it can be easily checked that $\tilde{G}^{-1} \notin \mathbb{H}_{s}$ for any $s$. This is why the theory above regarding the Helmholtz filters fails in this case, because it is based on the fact that $G$ defines an isomorphism between the $\mathbb{H}_{s}$ spaces.

However, the ADM may be considered for the Gaussian filter, and the resulting model yields a well posed problem [34]. Moreover, we shall show in what follows that it can be approximated in some sense by a sequence of operators which fall within the framework of the theory presented above. 
5.2. Approximation of the Gaussian filter. We note that for all $\mathbf{k} \in \mathcal{T}_{3}^{\star}$ fixed,

$$
\tilde{G}_{\mathbf{k}}=\lim _{m \rightarrow} \tilde{G}_{m, \mathbf{k}}, \quad \text { where } \quad \tilde{G}_{m, \mathbf{k}}=\left(1+\frac{\alpha^{2}|\mathbf{k}|^{2}}{24 m}\right)^{-m} .
$$

Let $\tilde{G}_{m}$ denote the kernel

$$
\tilde{G}_{m}(\mathbf{x})=\sum_{\mathbf{k} \in \mathcal{T}_{3}}\left(1+\frac{\alpha^{2}|\mathbf{k}|^{2}}{24 m}\right)^{-m} e^{i \mathbf{k} \cdot \mathbf{x}}
$$

which corresponds to the operator, still denoted by $\tilde{G}_{m}$,

$$
\tilde{G}_{m}=\left(1-\frac{\alpha^{2}}{24 m} \Delta\right)^{-m}
$$

In a sense that needs to be specified, the sequence $\left(\tilde{G}_{m}\right)_{m \in \mathbb{N}}$ converges to $\tilde{G}$. To be more specific, we have the following result.

Lemma 5.1. For all $\mathbf{k} \in \mathcal{T}_{3}^{\star}$,

$$
\left|\tilde{G}_{\mathbf{k}}-\tilde{G}_{m, \mathbf{k}}\right| \leq \frac{2}{m} \cdot 7
$$

Proof. We prove in Appendix A the technical inequality (A.7):

$$
\forall x \geq 0, \quad \forall m \geq 1, \quad\left|\left(1+\frac{x}{m}\right)^{-m}-e^{-x}\right| \leq \frac{2}{m} .
$$

We deduce inequality (5.8) upon replacing in this inequality $x$ by $\frac{\alpha^{2}|\mathbf{k}|^{2}}{24}$.

The following corollary is straightforward.

Corollary 5.2. For all $\mathbf{u} \in \mathbb{H}_{\mathbf{s}}$,

$$
\left\|\tilde{G} \mathbf{u}-\tilde{G}_{m} \mathbf{u}\right\|_{s} \leq \frac{2}{m}\|\mathbf{u}\|_{s}
$$

In other words, there is a weak star convergence of the sequence of operators $\left(\tilde{G}_{m}\right)_{m \in \mathbb{N}}$ to the Gaussian filter $\tilde{G}$ in $\mathbb{H}_{s}(s \geq 0)$.

5.3. Powers of the second order filter. In what follows we put, for $m$ fixed,

$$
\mu^{2}=\frac{\alpha^{2}}{24 m},
$$

and we denote by $H_{m}$ the $m^{\text {th }}$ power of the second order Helmholtz operator

$$
H_{m}=\left(\mathrm{I}-\mu^{2} \Delta\right)^{-m} \text {. }
$$

\footnotetext{
${ }^{7}$ This estimate is uniform in $\mathbf{k}$, but unfortunately we cannot conclude from this the normal convergence of the kernel sequence $\left(\tilde{G}_{m}\right)_{m \in \mathbb{N}}$ because the series $1 / m$ is not convergent.
} 
Estimating the modeling error that corresponds to $H_{m}$ yields estimates that correspond to $G_{m}$. The theory developed above regarding the Helmholtz operators applies to the operator $H_{m}$. Indeed, let

$$
\hat{H}_{m, \mathbf{k}}=\frac{1}{\left(1+\mu^{2}|\mathbf{k}|^{2}\right)^{m}}
$$

be the symbol of $H_{m}$. Using the scalar inequality $1+x^{m} \leq(1+x)^{m} \leq 2^{m-1}\left(1+x^{m}\right)$ for positive $x$, we get

$$
\frac{1}{2^{m-1}\left(1+\mu^{2 m}|\mathbf{k}|^{2 m}\right)} \leq \hat{H}_{m, \mathbf{k}} \leq \frac{1}{1+\mu^{2 m}|\mathbf{k}|^{2 m}}
$$

Using results of [4] (Section 6), we deduce from (5.13) that the ADM corresponding to $H_{m}$ has a unique regular weak solution $\left(\overline{\mathbf{u}}_{N}, \bar{p}_{N}\right)$, in the sense of Definition 2.2 with $p=m$. Furthermore, this sequence of solutions converges to some solution $(\overline{\mathbf{u}}, \bar{p})$ of the filtered NSE when $N$ goes to infinity. In the next theorem we estimate $\varepsilon_{N}$ corresponding to the new filter $H_{m}$.

Theorem 5.3. Let $\varepsilon_{N}=\overline{\mathbf{u}}-\overline{\mathbf{u}}_{N}$ be the modeling error corresponding to $H_{m}$, and assume that (3.1) still holds. Then we have ${ }^{8}$

$$
\begin{aligned}
& \left\|\varepsilon_{N}(t, \cdot)\right\|_{0}^{2}+\mu^{2 m}\left\|\varepsilon_{N}(t, \cdot)\right\|_{m}^{2}+\nu \int_{0}^{t}\left(\left\|\nabla \varepsilon_{N}(s, \cdot)\right\|_{0}^{2}+\mu^{2 m}\left\|\nabla \varepsilon_{N}(s, \cdot)\right\|_{m}^{2}\right) d s \\
\leq & \frac{8 C^{2} \mu m^{1 / 2}}{\nu(4(N+1))^{1 / 2 m}}\|\mathbf{u}\|_{L^{4}\left(\mathbf{H}_{1}\right)}^{4} e^{\frac{27}{2 \nu^{3}}\|\mathbf{u}\|_{L^{4}\left(\mathbf{H}_{1}\right)}^{4}} .
\end{aligned}
$$

Proof. Thanks to (5.13), one can copy line by line proofs of Theorem (3.2) and Corollary (3.1) and derive

$$
\begin{aligned}
& \left\|\varepsilon_{N}(t, \cdot)\right\|_{0}^{2}+\mu^{2 m}\left\|\varepsilon_{N}(t, \cdot)\right\|_{m}^{2}+\nu \int_{0}^{t}\left(\left\|\nabla \varepsilon_{N}(s, \cdot)\right\|_{0}^{2}+\mu^{2 m}\left\|\nabla \varepsilon_{N}(s, \cdot)\right\|_{m}^{2}\right) d s \\
\leq & \frac{2}{\nu} e^{\frac{27}{2 \nu^{3}}\|\mathbf{u}\|_{L^{4}\left(\mathbf{H}_{1}\right)}^{4}} \int_{0}^{t}\left\|\boldsymbol{\tau}_{N}(s, \cdot)\right\|_{0}^{2} d s .
\end{aligned}
$$

It remains to estimate $\left\|\boldsymbol{\tau}_{N}(s, \cdot)\right\|_{0}^{2}$. Step 4 in the proof of Lemma 4.1 can be reused, so that (4.5) still holds in this case. Therefore, we only have to bound

$$
\left\|\mathbf{u}-D_{N} \overline{\mathbf{u}}\right\|_{1 / 2}^{2}=\sum_{\mathbf{k} \in \mathcal{T}_{3}^{\star}}\left(1-\frac{1}{\left(1+\mu^{2}|\mathbf{k}|^{2}\right)^{m}}\right)^{2(N+1)}|\mathbf{k}|\left|\hat{\mathbf{u}}_{\mathbf{k}}\right|^{2},
$$

where as usual $\mathbf{u}=\sum_{\mathbf{k} \in \mathcal{T}_{3}^{\star}} \hat{\mathbf{u}}_{\mathbf{k}} e^{i \mathbf{k} \cdot \mathbf{x}}$. We apply the technical inequality (A.2) proved in Appendix A below, with $x=\mu|\mathbf{k}|, a=2(N+1)>1, m \geq 1$. We obtain

$$
\left(1-\frac{1}{\left(1+\mu^{2}|\mathbf{k}|^{2}\right)^{m}}\right)^{2(N+1)} \leq \frac{\sqrt{m} \mu}{(4(N+1))^{1 / 2 m}}|\mathbf{k}| .
$$

We multiply the result by $|\mathbf{k}|\left|\hat{\mathbf{u}}_{\mathbf{k}}\right|^{2}$ and get

$$
\left(1-\frac{1}{\left(1+\mu^{2}|k|^{2}\right)^{m}}\right)^{2 N+2}|\mathbf{k}|\left|\hat{\mathbf{u}}_{\mathbf{k}}\right|^{2} \leq \frac{\sqrt{m} \mu}{(4(N+1))^{1 / 2 m}}|\mathbf{k}|^{2}\left|\hat{\mathbf{u}}_{\mathbf{k}}\right|^{2},
$$

\footnotetext{
${ }^{8}$ The constant $C$ is as in Theorem 3.1, inequality (3.9); see also Lemma 4.1.
} 
hence

$$
\left\|\mathbf{u}-D_{N} \overline{\mathbf{u}}\right\|_{1 / 2}^{2} \leq \frac{\sqrt{m} \mu}{(4(N+1))^{1 / 2 m}}\|\mathbf{u}\|_{1}^{2}
$$

which yields by (4.5),

$$
\|\boldsymbol{\tau}\|_{0}^{2} \leq \frac{4 C^{2} \sqrt{m} \mu}{(4(N+1))^{1 / 2 m}}\|\mathbf{u}\|_{1}^{4}
$$

giving (5.14) thanks to (5.15).

5.4. Passing to the limit. From the results of Subsection 5.3, we deduce thanks to the relation (5.10) that the ADM associated to the filter specified in Subsection (5.3) has a unique solution $\left(\overline{\mathbf{u}}_{N, m}, \bar{p}_{N, m}\right)$ which converges to some solution $\left(\overline{\mathbf{u}}_{m}, \bar{p}_{m}\right)$, of the filtered NSE, by assuming that $\left(\mathbf{u}_{m}, p_{m}\right)$ satisfies the regularity assumption (3.1).

Let $\boldsymbol{\varepsilon}_{N, m}=\overline{\mathbf{u}}_{m}-\overline{\mathbf{u}}_{N, m}$ denote the corresponding modeling error. Thanks to (5.14), we obtain ${ }^{9}$

$$
\begin{gathered}
\left\|\boldsymbol{\varepsilon}_{N, m}(t, \cdot)\right\|_{0}^{2}+\alpha^{2 m}(24 m)^{-m}\left\|\boldsymbol{\varepsilon}_{N, m}(t, \cdot)\right\|_{m}^{2}+\nu \int_{0}^{t}\left(\left\|\nabla \boldsymbol{\varepsilon}_{N, m}(s, \cdot)\right\|_{0}^{2}\right. \\
\left.+\alpha^{2 m}(24 m)^{-m}\left\|\nabla \boldsymbol{\varepsilon}_{N, m}(s, \cdot)\right\|_{m}^{2}\right) d s \\
\leq \frac{2 C^{2} \alpha}{\nu(4(N+1))^{1 / 2 m}}\|\mathbf{u}\|_{L^{4}\left(\mathbf{H}_{1}\right)}^{4} e^{\frac{27}{2 \nu^{3}}\|\mathbf{u}\|_{L^{4}\left(\mathbf{H}_{1}\right)}^{4}}
\end{gathered}
$$

Without any convergence result regarding the ADMs associated to the Gaussian filter (5.1) when $N$ goes to infinity, we cannot consider the corresponding modeling error, and therefore take the limit in (5.21) when $m$ goes to infinity. Nevertheless, we observe that for a fixed $N$, the right hand side of (5.1) converges, as $m \rightarrow \infty$, to some $C=C(\nu, \alpha, \mathbf{u}, C)$, which does not depend on $N$. We can only deduce a bound for the sup limit of the terms in the right hand side.

\section{Conclusions and open problems}

6.1. Typical size of the constants. The main estimate (3.6) we get in the paper yields the rate of convergence to zero of the modeling error in the case of Helmholtz filter of order $p$. The bound involves a constant of the form

$$
\kappa=\frac{1}{\nu}\|\mathbf{u}\|_{L^{4}\left(\mathbf{H}_{1}\right)}^{4} e^{\frac{27}{2 \nu^{3}}\|\mathbf{u}\|_{L^{4}\left(\mathbf{H}_{1}\right)}^{4}}
$$

The number $N$ of iterations required to reduce substantially the SFS area is driven by the size of the constant $\kappa$.

This constant involves gradients of the true velocity of the fluid, which may be very large. For instance, in some turbulent boundary layer, one may observe flows for which $\nabla \mathbf{u}$ is of order $3.10^{4} \mathrm{~s}^{-1}$ in layers of thickness about $10^{-1} \mathrm{~m}$. For such an air layer at $50^{\circ}$ (that can be considered as incompressible) of width and length equal to $1 \mathrm{~m}$, over a time range of $1 \mathrm{~s}$, with $\nu \approx 20.10^{-6} \mathrm{~m}^{2} \mathrm{~s}^{-1}$, we find

$$
\kappa \approx 10^{10^{28}} \mathrm{~m}^{4} \mathrm{~s}^{-2},
$$

\footnotetext{
${ }^{9}$ For simplicity, we use $\mu m^{1 / 2} \leq \alpha / 4$ instead of (5.10).
} 
which is a very huge constant. Therefore, even if the resolution would be of order $\alpha=$ $10^{-18} \mathrm{~m}$, to fully solve such a flow, the number of iteration $N$ required to substantially reduce $\varepsilon_{N}$ is so large that the deconvolution algorithm seems not suitable for practical simulations, which is in contradiction with results of [38], suggesting that very few iterations are sufficient to significantly reduce the SFS area.

The rate of convergence as $(p(N+1))^{-1 / 4 p}$ comes from estimating norms of the residual stress $\tau_{N}$ involved in the equation for $\varepsilon_{N}$, whereas the constant $\kappa$ considered above comes from Gronwall's Lemma, which is known to lead to non optimal results. This yields the conjecture that the rate of convergence we found is optimal, which is not the case of the constant, that might be substantially improved. This conjecture is motivated by results in [38], already mentioned, and other practical simulations using high accuracy methods, such as pseudospectral methods, where people typically take $\mathrm{N}=5$ to $7[16]$.

Furthermore, there is also the question on how the regularity assumption $\mathbf{u} \in$ $L^{4}\left(\mathbf{H}_{1}\right)$ could be weakened.

REMARK 6.1. Let denote $\tilde{\kappa}$ the optimal constant, and let $\varepsilon_{0}$ be a given accuracy threshold. Observe that

$$
N=1+\frac{1}{p} e^{-p \log \left(\tilde{\kappa}^{-1} \varepsilon_{0}\right)}
$$

is the number of iterations necessary to achieve the required accuracy. This number is minimal when $p=p_{c}$,

$$
p_{c}=-\frac{1}{\log \left(\tilde{\kappa}^{-1} \varepsilon_{0}\right)},
$$

which might be less than 1 . This is why $p=1$ may not be the optimal choice.

6.2. Gaussian filter. The issue of convergence of ADM in the case of the Gaussian filter also remains. We conjecture that the convergence holds, but in a very weak sense as yet undefined, according to Corollary 5.2.

Appendix A. This technical appendix aims to provide two general inequalities that have been used in the proof of the estimate (4.9).

Theorem A.1. The scalar inequality

$$
\left(1-\frac{1}{(1+x)^{m}}\right)^{a} \leq \frac{m x}{\sqrt[m]{a}}
$$

holds true for any $x \geq 0, a, m \geq 1$.

Proof. We apply the Lagrange intermediate formula to the left hand side above (as a function of $x$ ) on $[0, x]$ and obtain the equivalent inequality

$$
a\left(1-\frac{1}{(1+x)^{m}}\right)^{a-1} \frac{1}{(x+1)^{m+1}} \leq \frac{1}{\sqrt[m]{a}}
$$

for any $x \geq 0, a, m \geq 1$.

We make the substitution $y=\frac{1}{1+x} \in(0,1)$ and the inequality becomes

$$
\left(1-y^{m}\right)^{a-1} y^{m+1} \leq a^{-1-1 / m}
$$


for any $y \in(0,1), a, m \geq 1$.

The left side above as a function of $y$ has maximum value on $(0,1)$ equal to $\left(\frac{a m-m}{a m+1}\right)^{a-1}\left(\frac{m+1}{a m+1}\right)^{\frac{m+1}{m}}$.

Therefore we need to show that

$$
\left(\frac{a m-m}{a m+1}\right)^{a-1}\left(\frac{m+1}{a m+1}\right)^{\frac{m+1}{m}} \leq a^{-1-1 / m}
$$

for $a, m \geq 1$.

Now let $z=1 / m \in(0,1]$. The inequality becomes

$$
\left(\frac{a-1}{a+z}\right)^{a-1}\left(\frac{1+z}{1+z / a}\right)^{1+z} \leq 1
$$

for any $a \geq 1, z \in(0,1]$.

Let $f$ be the function defined by the natural $\log$ of the left hand side above, i.e.

$$
f(a)=\ln \left(\left(\frac{a-1}{a+z}\right)^{a-1}\left(\frac{1+z}{1+z / a}\right)^{1+z}\right) .
$$

The derivative of $f$ is

$$
f^{\prime}(a)=\ln (a-1)-\ln (z+a)+\frac{1+z}{a} .
$$

The second derivative is

$$
f^{\prime \prime}(a)=-\frac{(z+1)(a z-z-a)}{a^{2}(a-1)(z+a)} .
$$

Obviously, because $a \geq 1$ and $z \in[0,1)$,

$$
f^{\prime \prime}(a) \geq 0
$$

We conclude that the first derivative is increasing, therefore

$$
f^{\prime}(a) \leq \lim _{a \rightarrow \infty} f^{\prime}(a)=0 .
$$

Therefore $f^{\prime}$ is negative, so $f$ is decreasing. It follows that

$$
f(a) \leq \lim _{a \rightarrow 1} f(a)=0 .
$$

We conclude that $f(a) \leq 0$, which proves the inequality.

Corollary A.2. The scalar inequality

$$
\left(1-\frac{1}{\left(1+x^{2}\right)^{m}}\right)^{a} \leq \frac{\sqrt{m} x}{\sqrt[2 m]{2 a}}
$$

holds true for any $x \geq 0, a, m \geq 1$. 
Proof. In the previous inequality we replace $x$ with $x^{2}$ and get

$$
\left(1-\frac{1}{\left(1+x^{2}\right)^{m}}\right)^{a} \leq \frac{m x^{2}}{\sqrt[m]{a}}
$$

for any $x \geq 0, a, m \geq 1$.

Replace in this inequality $a$ with $2 a$, but still keep $a \geq 1$ (but works for $a \geq 1 / 2$ ) to get

$$
\left(1-\frac{1}{\left(1+x^{2}\right)^{m}}\right)^{2 a} \leq \frac{m x^{2}}{\sqrt[m]{2 a}}
$$

for any $x \geq 0, a, m \geq 1$.

Now extract the square root of both sides to find

$$
\left(1-\frac{1}{\left(1+x^{2}\right)^{m}}\right)^{a} \leq \frac{\sqrt{m} x}{\sqrt[2 m]{2 a}}
$$

REmark A.1. Setting $m=1$ in the previous inequality gives

$$
\left(\frac{x^{2}}{1+x^{2}}\right)^{a} \leq \frac{x}{\sqrt{a}}
$$

for any $x \geq 0, a \geq 1$.

The following inequality will be used to approximate the Gaussian filter with a power of the second order Helmholtz filter and calculate the accuracy of this approximation.

Theorem A.3. The scalar inequality

$$
\left|(1+x / n)^{-n}-e^{-x}\right| \leq \frac{2}{n}
$$

is valid for any real $x \geq 0$ and any integer $n \geq 1$.

It is well-known that as a function of $n$ (and fixed $x \geq 0$ ) the expression

$$
(1+x / n)^{-n}
$$

is decreasing and converges to $e^{-x}$ as $n \rightarrow \infty$.

Therefore, the left hand side in (A.7) can be written as

$$
\left|(1+x / n)^{-n}-e^{-x}\right|=(1+x / n)^{-n}-e^{-x}=e^{-n \ln (1+x / n)}-e^{-x}=e^{-n \ln (1+y)}-e^{-n y},
$$

where $y=x / n \geq 0$.

Applying the intermediate value theorem of Lagrange (corresponding to the function $\xi \rightarrow e^{-n \xi}$ ) to the last term above we get that

$$
e^{-n \ln (1+y)}-e^{-n y}=n e^{-n \xi}(y-\ln (1+y))
$$

for some $\xi \in[\ln (1+y), y]$. Here we used $\ln (1+y) \leq y$ for $y \geq 0$. Because $e^{-n \xi} \leq$ $e^{-n \ln (1+y)}$, we further have that

$$
e^{-n \ln (1+y)}-e^{-n y} \leq n e^{-n \ln (1+y)}(y-\ln (1+y))=n(1+y)^{-n}(y-\ln (1+y))
$$


for any real $y \geq 0$ and integer $n \geq 1$

The term $y-\ln (1+y)$ appearing in the last term in the inequality above is estimated as

$$
0 \leq y-\ln (1+y) \leq \frac{y^{2}}{2}
$$

for any real $y \geq 0$

Going back to inequality (A.8) we finally have

$$
e^{-n \ln (1+y)}-e^{-n y} \leq n(1+y)^{-n} \frac{y^{2}}{2} .
$$

We replace $y=x / n$ and obtain

$$
\left(1+\frac{x}{n}\right)^{-n}-e^{-x} \leq n\left(1+\frac{x}{n}\right)^{-n} \frac{x^{2}}{2 n^{2}}=x^{2}\left(1+\frac{x}{n}\right)^{-n} \frac{1}{2 n} .
$$

But, as pointed out before, for any fixed $x$ the function $n \rightarrow(1+x / n)^{-n}$ is decreasing, so we have that, for $n \geq 2$,

$$
\left(1+\frac{x}{n}\right)^{-n} \leq\left(1+\frac{x}{2}\right)^{-2} \leq \frac{4}{1+x^{2}} .
$$

Therefore, for $n \geq 2$,

$$
\left(1+\frac{x}{n}\right)^{-n}-e^{-x} \leq x^{2}\left(1+\frac{x}{n}\right)^{-n} \frac{1}{2 n} \leq \frac{4 x^{2}}{1+x^{2}} \frac{1}{2 n} \leq \frac{2}{n} .
$$

For $n=1$ the left hand side of (A.7) becomes

$$
(1+x)^{-1}-e^{-x} \leq(1+x)^{-1}+e^{-x} \leq 2
$$

for any $x \geq 0$, so the inequality (A.7) is valid for $n=1$ as well.

Acknowledgment. The authors would like to thank Prof. Leo Rebholz who carefully reviewed this paper.

\section{REFERENCES}

[1] Maxima, a computer algebra system, http://maxima.sourceforge.net/, Version 5.18.1, 2009.

[2] N.A. Adams and S. Stolz, Deconvolution methods for subgrid-scale approximation in largeeddy simulation, in B. Geurts (ed.), Modern Simulation Strategies for Turbulent Flow. R.T. Edwards, 21-41, 2001.

[3] J. Bardina, J.H. Ferziger, and W.C. Reynolds, Improved subgrid scale models for large eddy simulation, AIAA paper, 80, 1357, 1980.

[4] L. Berselli and R. Lewandowski, Convergence of approximate deconvolution models to the mean Navier-Stokes equations, Annales de l'Institut Henri Poincare (C), Non Linear Analysis, 29, 171-198, 2012.

[5] L.C. Berselli, T. Iliescu, and W.J. Layton, Mathematics of Large Eddy Simulation of Turbulent Flows, Sci. Comput. Springer-Verlag, Berlin, 2006.

[6] Y. Cao, E.M. Lunasin, and E.S. Titi, Global well-posedness of the three-dimensional viscous and inviscid simplified Bardina turbulence models, Comm. Math. Sci., 4(4), 823-848, 2006.

[7] F.K. Chow, R.L. Street, M. Xue, and J.H. Ferziger, Explicit filtering and reconstruction turbulence modeling for large-eddy simulation of neutral boundary layer flow, J. Atmos. Sci., 62(7), 2058-2077, 2005. 
[8] A. Dunca, A two-level multiscale deconvolution method for the large eddy simulation of turbulent flows, Math. Models Meth. Appl. Sci., 22(06), 1250001, 2012.

[9] A. Dunca and Y. Epshteyn, On the Stolz-Adams deconvolution model for the large-eddy simulation of turbulent flows, SIAM J. Math. Anal., 37(6), 1890-1902 (electronic), 2006.

[10] J.W. Eaton, GNU Octave Manual, Network Theory Limited, 2002.

[11] M. Germano, Fundamentals of Large Eddy Simulation, in R. Peyret and E. Krause, (eds.), CISM Courses and Lectures, Advanced Turbulent flow computations, Springer, 395, 2000.

[12] T.H. Gronwall, Note on the derivative with respect to a parameter of the solutions of a system of differential equations, Ann. Math., 20(4), 292-296, 1919.

[13] J. Gullbrand and F.K. Chow, The effect of numerical errors and turbulence models in largeeddy simulation of channel flow, with and without explicit filtering, J. Fluid Mech., 495, 323-341, 2003.

[14] T. Iliescu and J. Borggaard, Approximate deconvolution boundary conditions for large eddy simulation, Appl. Math. Lett., 19(8), 735-740, 2006.

[15] W. Layton, Superconvergence of finite element discretization of time relaxation models of advection, BIT, 47(3), 565-576, 2007.

[16] W. Layton, Private Communication, 2013.

[17] W. Layton and R. Lewandowski, A simple and stable scale similarity model for large scale eddy simulation: Energy balance and existence of weak solutions, Appl. Math. Lett., 16, 1205-1209, 2003.

[18] W. Layton and R. Lewandowski, On a well-posed turbulence model, Disc. Cont. Dyn. Sys. Ser. B, 6(1), 111-128, 2005.

[19] W. Layton and R. Lewandowski, Residual stress of approximate deconvolution models of turbulence, J. Turbul., 7, 1-21, 2006.

[20] W. Layton and M. Neda, A similarity theory of approximate deconvolution models of turbulence, J. Math. Anal. Appl., 333(1), 416-429, 2007.

[21] W. Layton and M. Neda, Truncation of scales by time relaxation, J. Math. Anal. Appl., 325(2), 788-807, 2007.

[22] W. Layton and I. Stanculescu, K-41 optimised approximate deconvolution models, Inter. J. Comput. Sci. Math., 1(2-4), 396-411, 2007.

[23] W. Layton and I. Stanculescu, Chebychev optimized approximate deconvolution models of turbulence, Appl. Math. Comput., 208(1), 106-118, 2009.

[24] W.J. Layton and R. Lewandowski, On a well-posed turbulence model, Disc. Cont. Dyn. Sys. Ser. B, 6(1), 111-128 (electronic), 2006.

[25] W.J. Layton and R. Lewandowski, Residual stress of approximate deconvolution models of turbulence, J. Turbul., 7, 1-21, 2006.

[26] W.J. Layton and L. Rebholz, Approximate Deconvolution Models of Turbulence Approximate Deconvolution Models of Turbulence, Springer, Heidelberg, 2012.

[27] K.L. Lele, Compact finite different schemes with spectral-like resolution, J. Comput. Phys., 103, 16-42, 1992.

[28] R. Lewandowski, On a continuous deconvolution equation for turbulence models, Lecture Notes of Necas Center for Mathematical Modeling, 3, 69-102, 2009.

[29] R. Lewandowski, Approximations to the Navier-Stokes Equations, in preparation, release scheduled middle 2014.

[30] J.L. Lions and E. Magenes, Problèmes aux limites non homogènes et applications. Vol. 1, Travaux et Recherches Mathématiques, Dunod, Paris, 17, 1968.

[31] C.C. Manica and S. Merdan, Finite element error analysis of a zeroth order approximate deconvolution model based on a mixed formulation, J. Math. Anal. Appl., 331(1), 669-685, 2007.

[32] L. Rebholz, Conservation laws of turbulence models, J. Math. Anal. Appl., 326(1), 33-45, 2007.

[33] P. Sagaut, Large eddy simulation for incompressible flows, Sci. Comput., Springer-Verlag, Berlin, 2001.

[34] I. Stanculescu, Existence theory of abstract approximate deconvolution models of turbulence, Annali dell'Universita di Ferrara, 51(1), 145-168, 2008.

[35] I. Stanculescu and C.C. Manica, Leray-Tikhonov deconvolution models of fluid motion, Comput. Math. Appl., 60(5), 1440-1456, 2010.

[36] E. Stein and R. Shakarchi, Fourier Analysis: An Introduction, Princeton University Press, 2003.

[37] S. Stolz and N.A. Adams, An approximate deconvolution procedure for large-eddy simulation, Phys. Fluids, 11(7), 1699-1701, 1999.

[38] S. Stolz, N.A. Adams, and L. Kleiser, An approximate deconvolution model for large-eddy simulation with application to incompressible wall-bounded flows, Phys. Fluids, 13(4), 997- 
$1015,2001$.

[39] S. Stolz, N.A. Adams, and L. Kleiser, An approximate deconvolution model for large-eddy simulation with application to incompressible wall-bounded flows, Phys. Fluids, 13(4), 9971015, April 2001.

[40] S. Stolz, N.A. Adams, and L. Kleiser, The approximate deconvolution model for large-eddy simulations of compressible flows and its applications to shock-turbulent-boundary-layer interaction, Phys. Fluids, 13(10), 2985-3001, October 2001.

[41] R. Temam, Navier-Stokes Equations, AMS Chelsea Publishing, Providence, RI, 2001. Theory and Numerical Analysis, Reprint of the 1984 Edition.

[42] J. Weickert, Anisotropic Diffusion in Image Processing, ECMI Series, Teubner-Verlag, Stuttgart, Germany, 1998.

[43] A.P. Witkin, Scale-space filtering, Proc. Eighth Int. Joint Conf. on Artificial Intelligence, 2, 1019-1022, 1983. 